\title{
Plasmon-enhanced Fields and Upconversion Luminescence utilizing Earth-abundant Plasmonic Nanoparticles
}

\author{
Akorede Kalejaiye \\ Kalejaiye.jciunilorin@gmail.com
}

\begin{abstract}
This short review paper describes how earth-abundant plasmonic nanoparticle (specifically Magnesium, Aluminium and Sodium nanoparticles), which have not been given much attention possess advantages over conventional plasmonic particles, and can be used to achieve efficient upconversion luminescence - the enhancement of low energy excitations - due to their availability and stability. They can be used to achieve high upconversion luminescence efficiency by coupling their plasmonic resonances with the upconversion particles due to their localized field enhancement effect.

Copper nanoparticles just like any other plasmonic nanoparticles can be used for Metal Enhanced Fluorescence. A major advantage of Earth abundant nanoparticles is that they are more stable and readily available to be used in industrial applications for example in photovoltaic cells.
\end{abstract}




\section{Introduction}

Conventional plasmonic materials like $\mathrm{Au}$ and $\mathrm{Ag}$ are rare and unstable, they also pose financial constraints for real world technological applications of plasmonic materials. Plasmonic materials have promising applications in fields that range from agriculture to bioimaging because of their ability to manipulate light (since they have smaller wavelength) due the presence of Surface Plasmon Resonance which gives a desirable property known as upconversion luminescence that enables conversion of low energy excitations into high energy emissions but with the challenges posed by rare materials like Au and Ag nanoparticles (NP), we need to study upconversion luminescence in alternative plasmonic materials (like $\mathrm{Al}, \mathrm{Mg}, \mathrm{Cu}$ and $\mathrm{Na}$ ) which are more accessible.

Earth-abundant metals are majorly transition metals like copper and iron (also inexpensive metals like Aluminium and Magnesium) which are stable, have multiple valences and form coloured complexes and are readily available for large-scale industrial applications.

Plasmonic properties (LSPR) have been observed in metals such as $\mathrm{Mg}$ and $\mathrm{Al}$ that have been catalysed with Platinum-group metals, but LSPR in these nanoparticles depend on the size and shape of the nanoparticles and also the level of doping with platinum group metals.

Size of nanoparticles, determined during the fabrication process, affects the peak and bandwidth of SPR, when the particle size decreases from 20 to $12 \mathrm{~nm}$ a blue shift (due to radiative depolarization) in the band occurs, but from a 12 nm diameter, a red shift occurs. 


\section{LSPR in Earth Abundant Metal Nanoparticles}
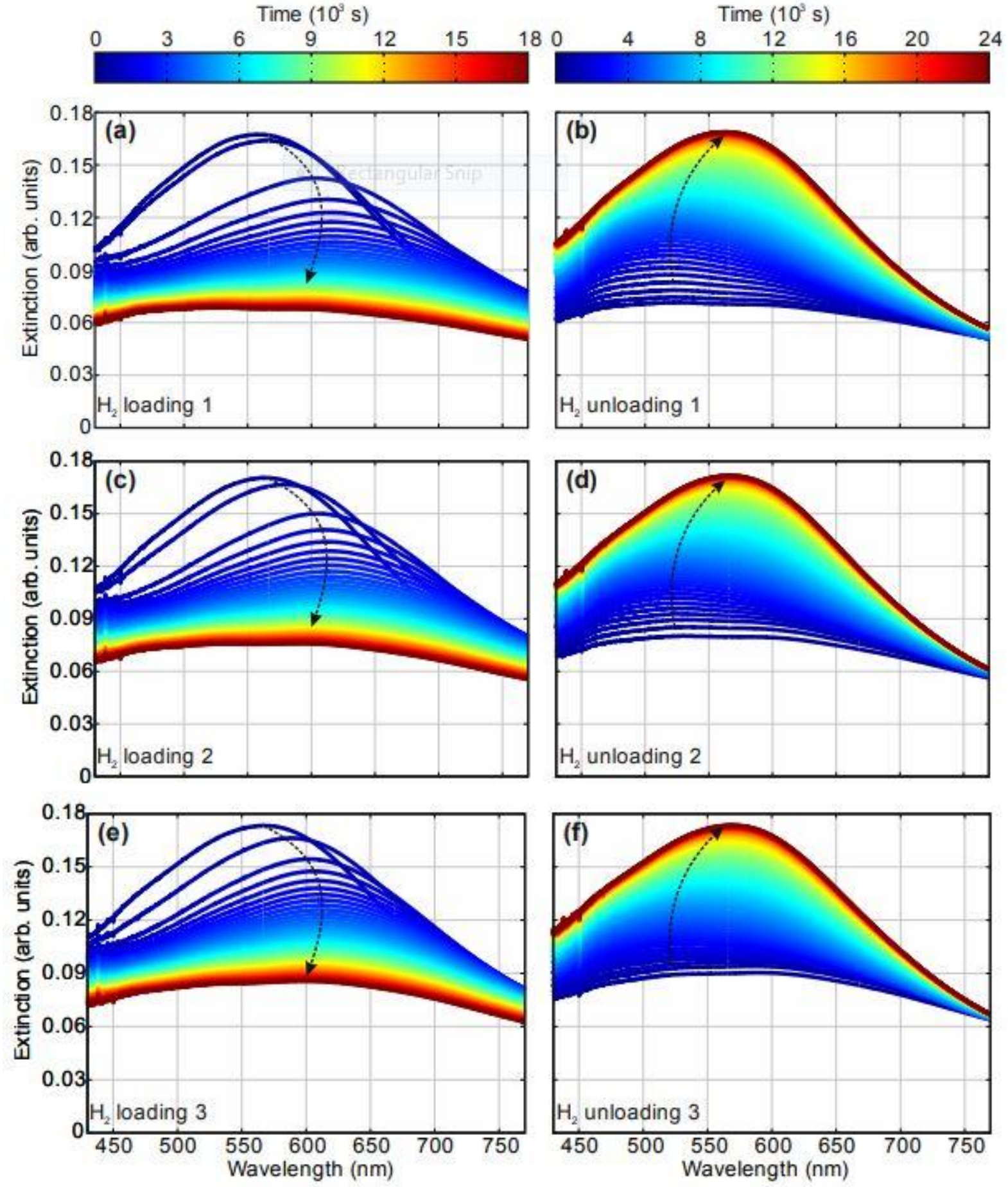

Fig. 1. Experimental Spectra of $\mathrm{H}_{2} / \mathrm{O}_{2}$ cycles in $\mathrm{Mg} / \mathrm{Ti} / \mathrm{Pd}$ disks - excitation of hydrogen/oxygen is used to describe plasmonics in the visible wavelength range. Adapted from [[3]] with permission from (C) 2018 American Chemical Society. 


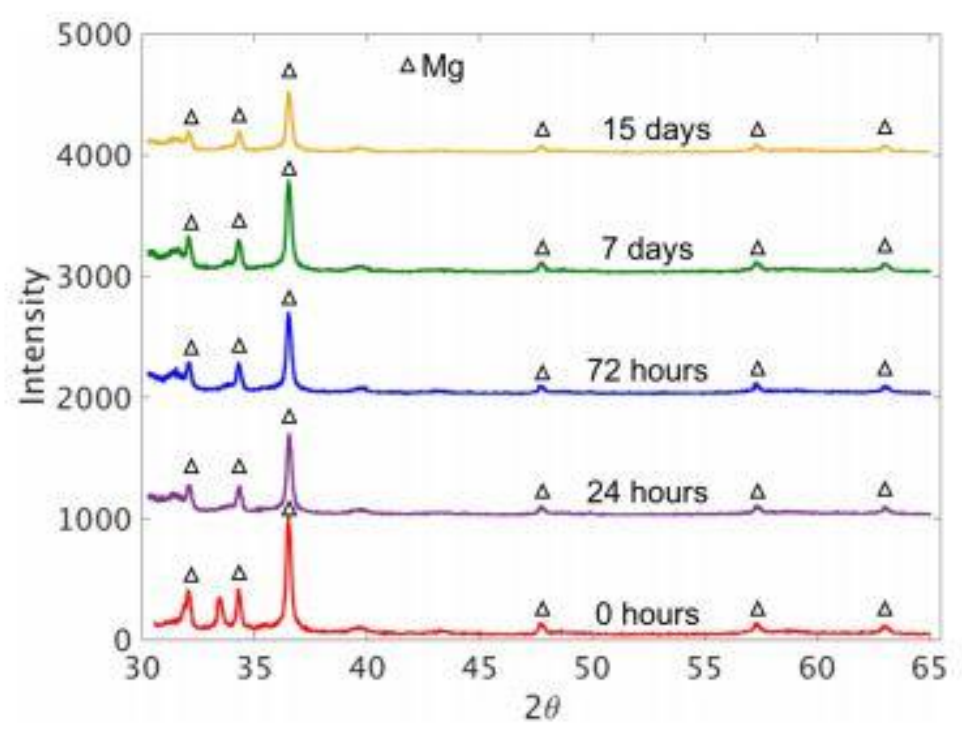

Fig. 2. Powder Xray diffraction pattern of Mg nanoparticles exposed to air. Adapted from [[4]] with permission from (c) 2018 American Chemical Society.

Several factors affects plasmonics in earth-abundant metal nanoparticles, the resonance frequency of plasmonic resonance in metallic nanopartcles is dictated by its geometry, this resonance also depend upon composition of the earth abundant metal nanoparticles. For instance, magnesium nanoparticle is capable of sustaining resonances across UV and NIR spectrum, tuning the shapes and sizes of magnesium NPs allows manipulation of local electric fields and resonant energies across different spectra.

E. Ringe et. al 2010, used highly resolved transmission electron microscopy LSPR method on one partcle to demonstrate the effect of geometry and composition, and substrate index of refraction on the plasmonic properties of some nanostructures and also changing its form or other factors causes a change to the plasmon band. The plasmonic resonance depends on the index of refraction of the medium and therefore the particle according to:

$$
\lambda_{\max }=\lambda_{\mathrm{p}} \sqrt{ } 2 \eta_{\mathrm{m}}{ }^{2}+1
$$

wavelength of LSPR inside a condensed matter is denoted by $\lambda_{\mathrm{p}}$, and $\eta_{\mathrm{m}}$ is the index of refraction of the medium. 
The fabrication technique of nanoparticles is vital because it dictates the dimensions and morphology which plays a part in realisation of plasmonic resonances in metals. Another factor which will alter plasmonic resonance band is the temperature. Reports have shown that SPR peak broadens and the intensity decreases after increasing temperature from 1.5 to $300 \mathrm{~K}$ for Gold and Silver nanoparticle clusters on a glass matrix, increasing temperature also correspond to decrease in density of free-electrons (as a result radiation damping causes lowered plasma frequency) and NP volume increase, that is why there is a shift and widening of SPR peak. [[2]]

The nature of the medium (with ion exchange) where NPs are present can alter SPR band position by affecting the Fermi level. In core-shell structures, where the properties (affected by core to shell ratio) change from the original properties of the constituent nanoparticles, an outer layer is used to modify the surface, optical or magnetic properties of an inner core particle which is done by either coating the nanoparticles on a preformed core or growing alternating layers on a surface modified nanoparticle. Plasmonic NP like magnesium can be used together with carbon as outer shell to protect magnetic cores made from iron from oxidation and corrosion, dye mixed with silica shell used to coat magnetic iron oxide cores helped enhance photoluminescence in the shell (at the same time improving magnetism from the core), also coating silica core with a gold shell helps modify position of surface Plasmon resonance.

\section{Upconversion Luminescence and Fluorescence}

Upconversion luminescence is an effect in which photons of low energy excitations produce high energy emissions. Upconversion nanoparticles which convert these low energy photons into high energy emissions have attract interest from scientists because of some of their unique photophysical characteristics like long luminescence lifetime and resistance to photobleaching. These properties have made them candidates for applications in fields such as display technologies, biomedical imaging, lightsource and solar energy harvesting.

Lanthanide doping of upconversion nanoparticles have been the major process of achieving upconversion luminescence in nanoparticles, since the Lanthanide ions (where electronic transitions occur in the $4 \mathrm{f}$ electron shells) acts as a sensitizer ion for activator ion of UCNP, Earth-abundant metal $\mathrm{Na}$ is sometimes 
utilized as a component of the core shell structure for improving enhancement ability of upconversion nanoparticles. The problem of low upconversion luminescence efficiency, the quality of the intensity of upcoversion luminescence, leads to a number of approaches for overcoming the barrier, the most promising of which is the enhancement of electric field by plasmonics [[6]]. In this method, plasmons (LSPR) are utilized to enhance absorption, emission and energy transfer processes occurring during the upconversion.

Photoactive complexes formed from ligands and Earth-abundant metals also have long lived excitations due to their photophysical properties [[15]][[16]] which can be exploited in optical research. L. A. Büldt and O. S. Wenger described the use of chelating diisocyanide ligands to enable $\operatorname{Mo}(0)$ and $\operatorname{Cr}(0)$ complexes with $\mathrm{d}^{6}$ electron configuration exhibit photophysical properties common with $\mathrm{Ru}(\mathrm{II})$ polypyridines or cyclometalated $\mathrm{Ir}$ (III) complexes (metalligand charge transfer). Some research papers have shown how Salen (and similar ligands) and its metal complexes can act as fluorophores for probe molecules due to its ability to carry oxygen and how traces of elements, cyanide and peroxides can be discovered through fluorescence property of Salen, its derivatives, and its complexes [[2]]. This property makes it easy for Salen complex of Manganese to detect DNA traces in solutions [[44]] through hypochromic shift in the ultraviolet absorption spectra.

This property can be utilized in metal enhanced fluorescence (MEF), MEF is similar to upconversion luminescence in that they take advantage of SPR from metals to transfer energy needed for enhancement of optical excitations. S. Pawar et. al 2019 reported the use of Au NPs for enhancement of fluorescence intensity in bipyridine-based construct 4-(pyridine-2-yl)-3H-pyrrolo[2,3-c] quinoline (PPQ) after binding with $\mathrm{Zn}^{2+}[[19]]$. Fares et al reported enhancement of photoluminescence of Erbium (III) ions in tellurite using plasmonic resonance.

\section{Plasmon-enhanced Upconversion Luminescence and Fluorescence Enhancement}

The "plasmon enhancement effects on UCL involve various processes including the absorption, emission, and energy transfer processes, as described below. Briefly, they are the (1) absorption enhancement of the sensitizer through the 
localized optical field, (2) modulation of the decay rate, emission intensity, spectral profile, and quantum yield via the Purcell effect, and (3) enhanced energy transfer process from the donors to acceptors" J. Liu et al, 2018 [[6]]. The most reported method of Plasmon-enhanced upconversion is by the enhancement of energy transfer, which is obtained through the interaction of free electrons in the acceptor of metallic structures and upconversion nanoparticles.

Plasmon enhanced upconversion can also be realised by overlapping LSPR with the upconversion emission wavelength in the plasmonic upconversion nanoparticle system.

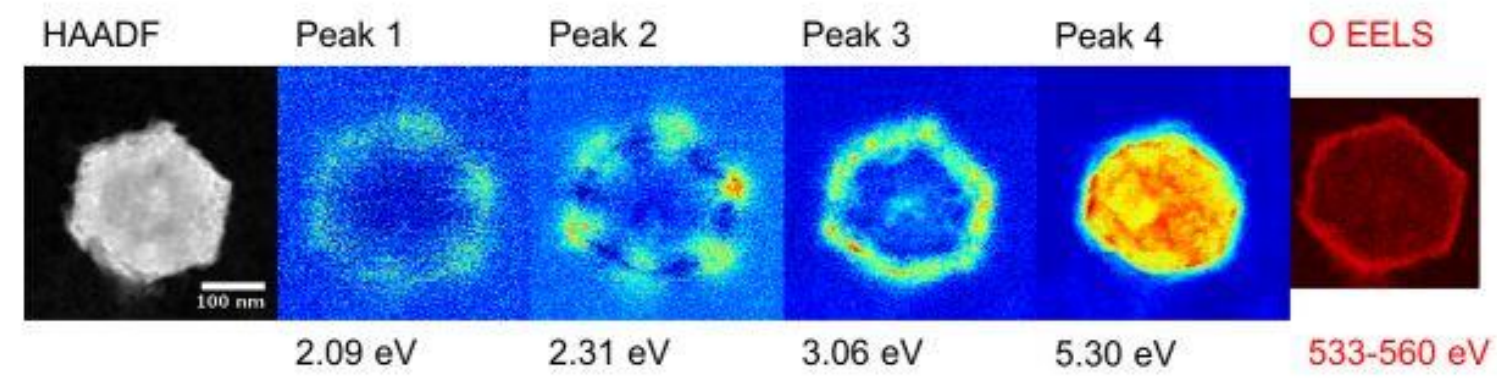

Fig 3. HAADF-STEM, SYEM-EELS maps of Plasmon modes from Gaussian fitting and STEM-EELS map of oxygen for a $284 \mathrm{~nm} \mathrm{Mg} \mathrm{NP}$ obtained over three months post synthesis. Adapted from [[4]] with permission from (C) 2018 American Chemical Society.

Since LSPR effect enables enhancement of local field in the region of the nanoparticles, periodic structures can be used to provide well defined coupling conditions for the irradiating light to achieve the absorption enhancement, therefore increasing absorption of sensitizer ions and also enhancing the emission intensity of plasmonic upconversion nanoparticles can lead to enhancement of upconversion luminescence by matching their combined plasmonic resonance with a field excitation. This concept is similar to effects in Metal Enhanced Fluorescence; the lightening rod effect where enhanced absorption supports enhanced emission and radiation of coupled fluorescence by surface plasmons induced in metals by fluorophores (Surface-PlasmonCoupled Emission). In the latter, the intensity of enhancement is determined by the metallic nanostructure's geometry, this can also be affected by non-radiative dissipative losses in metals (ohmic loss etc.) as they can lead to quenching of the enhancement. 
Increasing the efficiency of emission through the scattering of nanoparticles and improving the radiative decay rate of close fluorophores with a change in the fluorescence quantum yield and lifetime, and also increasing the near field around the fluorophore helps improve Metal Enhanced Fluorescence.

Studies reported the use of silver island films (SIF) and other silver surfaces, which can be explained using radiating Plasmon mode, to improve the intensity and photostability of fluorophores having low quantum yield based on their modes of interaction with the fluorophores. [[37]][[37]][[39]][[39]][[41]]

\section{Utilizing Earth Abundant Nanoparticles in Metal Enhanced Fluorescence and Plasmon-enhanced Upconversion Luminescence and Fields}

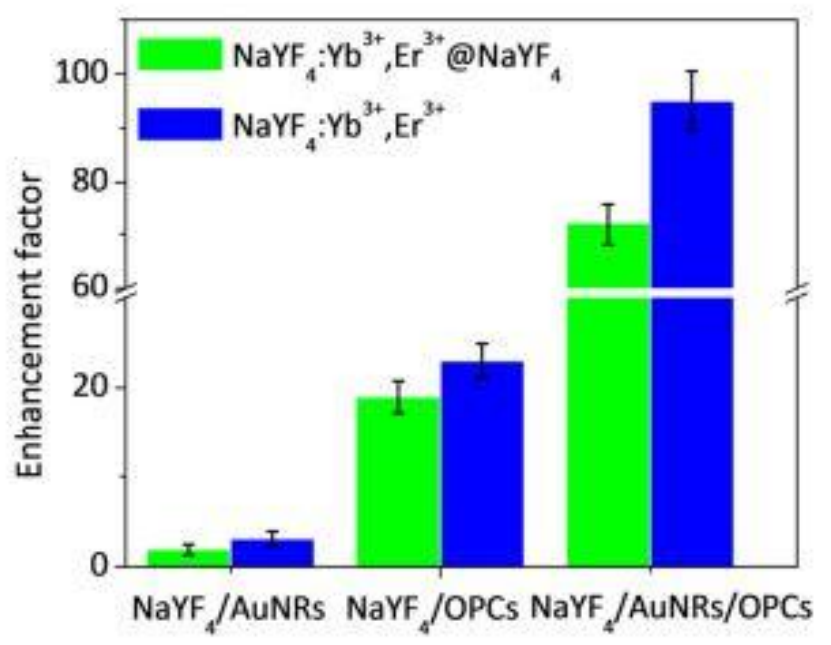

Fig 4. Enhancement factor for $\mathrm{UCL}$ of $\mathrm{NaYF}_{4} / \mathrm{Au}$ nanorods, $\mathrm{NaYF}_{4} / \mathrm{Opal}$ photonic crystals (OPCs), $\mathrm{NaYF}_{4} / \mathrm{Au}$ nanorods/OPCs with/without core-shell structures. Adapted from [[11]] with permission from @ 2016 John Wiley and Sons.

Enhancement of upconversion emission by plasmonics would not only increase emission efficiency but increase the rate of radiative decay - as described in Figure 4 above, enhancement of the $\mathrm{NaYF}_{4}$ hybrids depend on SPR between $\mathrm{NaYF}_{4}$, opal photonic crystals and Au NRs, and not dependent on the structure of $\mathrm{NaYF}_{4}$. Earth abundant metals $\mathrm{Na}$ and $\mathrm{Al}$ used in $\mathrm{NaYF}_{4}, \mathrm{SiO}_{2}$ and $\mathrm{Al}_{2} \mathrm{O}_{3}$ shells respectively, which are used as spacers to achieve emission enhancement in the core-shell plasmonic upconversion nanoparticle structures since metallic 
nanoparticles like Au NP shell attached to upconversion NP surface may also suppress the emission due to quenching. Quenching is suppression of radiative emission of photon from an atom close to a nanoparticle, it is the opposite of enhancement which is described by a factor known as quantum yield.

As plasmonic nanoantennas, Al plasmonic nanostructures are advantgeous over conventional metal nanostructures because they can reach parts of the electromagnetic spectrum requiring short-wavelength (plasmon resonance of Aluminium nanoparticles can extend to the ultraviolet spectrum because $\mathrm{Al} d-$ band lies above the $\mathrm{Al} \mathrm{Fermi} \mathrm{energy} \mathrm{band)} \mathrm{and} \mathrm{also} \mathrm{because} \mathrm{they} \mathrm{are} \mathrm{compatible}$ with complementary metal-oxide-semiconductors. These devices can focus radiation in to the sub-diffraction limit also couple out stored energy, making them emitting antennas, which helps control and visualize the optical field on the nanometre scale.

For solar cell designs with plasmonic cavities containing low minority carrier lifetime semiconductor, Al NPs are also utilized in metallic thin film materials with coaxial holes used as nanoantennas to realise field enhancement by fifty folds [[2]]. Al was also used in a "V-modelled antenna with two coherent pointdipole sources described in a study that showed the origin of unidirectional scattering of light as the phase and amplitude matching occurring at the Fano interference between two localized surface plasmon modes in a V-shaped NP" J. Jana et al 2016 [[2]].

$\mathrm{Cu}$ nanoparticles can be used to strongly enhance the Raman scattering intensity from particles absorbed on its surface, due to its plasmonic nature, a term known as Surface Enhanced Raman Scattering (SERS).

$\mathrm{Cu}$ nanoparticles just like $\mathrm{Au}$ and $\mathrm{Ag}$ nanoparticles exhibit Metal Enhanced Fluorescence (MEF), but $\mathrm{Ag}$ is used more because the imaginary parts in the dielectric function, which the absorption cross section rely on, is more favourable as compared to the others for example $\mathrm{Cu}$ where a close distance to the fluorophore causes its enhanced emission to quench. But $\mathrm{MEF}$ in $\mathrm{Cu}$ is based on scattering of its NP for increasing the coupling efficiency of the fluorescence emission to the far field in smaller NP $(100 \mathrm{~nm})$ with a high aspect ratio, leading to a decrease in the effect of ohmic losses on MEF. 


\section{Conclusion}

As earth-abundant metals like Magnesium and Aluminium continue to reveal exciting plasmonic properties, it is clear that they can rival conventional plasmonic materials like Gold and Silver in certain areas due to their stability, cost of acquiring for large scale production (as compared to precious metals $\mathrm{Au}$ and $\mathrm{Ag}$ ) and plasmonic activities in the visible light, Ultraviolet and Infrared ranges. They have also proven to be very useful in the areas of Plasmon enhanced upconversion, far field enhancement and metal enhanced fluorescence, which this paper focuses on.

$\mathrm{Cu}$ proves useful as a candidate for surface-plasmon-coupled emission in metal enhanced fluorescence due to the scattering of its NP and also in surface enhanced Raman scattering - a method used to detect presence of biomolecules of low abundance in biofluids. Al, another earth abundant metal is a great candidate for field enhancement as it can serve as a nanoantenna for absorbing and focusing light into a sub-diffraction limited volume and also control and visualize the optical field on the nanometre scale. Other earth abundant metals like $\mathrm{Na}$ are used in core-shell structures which depresses quenching in plasmonic upconversion nanoparticles. Al is very useful in many areas of research because of its plasmonics in the UV and IR range.

Properties like size, shape, Fermi level, fabrication technique used to create them and the nature of the medium they are placed in dictates the plasmonic effects in earth abundant metal nanoparticles and others alike.

\section{Acknowledgment}


I would like to give thanks to God for His grace and I acknowledge the American Chemical Society and John Wiley and Sons for allowing me to re-use the images in this review paper.

\section{Glossary of Terms}

HAADF: High angle annular dark field

STEM: Scanning transmission electron microscopy

EELS: Electron-energy loss spectroscopy

NP: Nanoparticle

LSPR: Localized Surface Plasmon Resonance

SPP: Surface Plasmon Polariton

SPR: Surface Plasmon Resonance

HRTEM: Highly Resolved Transmission Electron Microscopy

MEF: Metal Enhanced Fluorescence 


\section{References}

[1]R. Devasenathipathy, D. -Y. Wu and Z. -Q. Tian, Surface Plasmon Enhanced Chemical Reactions on Metal Nanostructures, Nanoplasmonics, 2019, doi: http://dx.doi.org/10.5772/intechopen.89606

[2] J. Jana, M. Gangulyb and T. Pa, Enlightening surface plasmon resonance effect of metal nanoparticles for practical spectroscopic application, $R S C$ $A d v ., 2016,6,86174$

[3]F. Sterl, N. Strohfeldt, R. Walter, R. Griessen, A. Tittl, and H. Giessen, Magnesium as Novel Material for Active Plasmonics in the Visible Wavelength Range, Nano Lett., 2015, 15, 12, 7949 -7955; doi: 10.1021/acs.nanolett.5b03029

[4] J. S. Biggins, S. Yazdi, E. Ringe, Magnesium Nanoparticle Plasmonics, Nano Lett. (2018) 18, 3752. https://doi.org/10.1021/acs.nanolett.8b00955

[5]P. Christopher, H. Xin and S. Linic, Visible-light-enhanced catalytic oxidation reactions on plasmonic silver nanostructures, Nature Chemistry volume 3, 467-472(2011). https://doi.org/10.1038/nchem.1032

[6] J. Liu, H. He, D. Xiao, S. Yin, W. Ji, S. Jiang, D. Luo, B. Wang, and Y. Liu, Recent Advances of Plasmonic Nanoparticles and their Applications, Materials 2018, 11, 1833; doi:10.3390/ma11101833

[7]E. Townsend et al, Approaching the quantum limit for nanoplasmonics, $J$. Mater. Res., Vol. 30, No. 16, Aug 28, 2015; doi: 10.1557/jmr.2015.232

[8] A. Nadort, J. Zhao, E. M. Goldys, Lanthanide upconversion luminescence at the nanoscale: fundamentals and optical properties, Nanoscale, 2016, 8, 13099-13130; doi: 10.1039/C5NR08477F

[9]C. Duan, L. Liang, L. Li, R. Zhang, Z. P. Xu, Recent progress in upconversion luminescence nanomaterials for biomedical applications, $J$. Mater. Chem. B, 2018, 6, 192-209; https://doi.org/10.1039/C7TB02527K

[10] Yin, Z.; Li, H.; Xu, W.; Cui, S.B.; Zhou, D.L.; Chen, X.; Zhu, Y.S.; Qin, G.S.; Song, H.W. Local field modulation induced three-order upconversion enhancement: Combining surface plasmon effect and 
photonic crystal effect. Adv. Mater. 2016, 28, 2518-2525; https://doi.org/10.1002/adma.201502943

[11] S. Hu, H. Cao, X. Wu, S. Zhan, Q. Wu, Z. Tang, Y. Liu, Upconversion Luminescence and Magnetic Turning of $\mathrm{NaLuF}_{4}: \mathrm{Yb}^{3+} / \mathrm{Tm}^{3+} / \mathrm{Gd}^{3+}$ Nanoparticles and Their Application for Detecting Acriflavine, Journal of Nanomaterials, vol. 2016, Article ID 2463479, 9 pages, 2016. https://doi.org/10.1155/2016/2463479

[12] L. Zhao, J. Peng, M. Chen, Y. Liu, L. Yao, W. Feng, F. Li, YolkShell Upconversion Nanocomposites for LRET Sensing of Cysteine/Homocysteine, ACS Appl. Mater. Interfaces 2014, 6, 14, 1119011197. https://doi.org/10.1021/am501249p

[13] Christopher, P., Xin, H. \& Linic, S., Visible-light-enhanced catalytic oxidation reactions on plasmonic silver nanostructures. Nature Chem 3, 467-472 (2011). https://doi.org/10.1038/nchem.1032

[14] B. H. Zhou, J. D. Rinehart, A Size Threshold for Enhanced Magnetoresistance in Colloidally Prepared $\mathrm{CoFe}_{2} \mathrm{O}_{4}$ Nanoparticle Solids, ACS Cent. Sci. 2018, 4, 9, 1222-1227, https://doi.org/10.1021/acscentsci.8b00399

[15] O. S. Wenger, Photoactive Complexes with Earth-Abundant Metals, J. Am. Chem. Soc. 2018, 140, 42, 13522-13533. https://doi.org/10.1021/jacs.8b08822

[16] L. A. Büldt, O. S. Wenger, Luminescent complexes made from chelating isocyanide ligands and earth-abundant metals, Dalton Trans., 2017, 46, 15175-15177. https://doi.org/10.1039/C7DT03620E

[17] Wen, S., Zhou, J., Zheng, K. et al. Advances in highly doped upconversion nanoparticles. Nat Commun 9, $2415 \quad$ (2018). https://doi.org/10.1038/s41467-018-04813-5

[18] Ai-Hua Li and Guanying Chen, Controlling lanthanide-doped upconversion nanoparticles for brighter luminescence, 2020 J. Phys. D: Appl. Phys. 53043001

[19] S. Pawar, A. Bhattacharya, A. Nag, Metal-Enhanced Fluorescence Study in Aqueous Medium by Coupling Gold Nanoparticles and Fluorophores Using a Bilayer Vesicle Platform, ACS Omega 2019, 4, 3, 5983-5990. https://doi.org/10.1021/acsomega.9b00036

[20] C. Cao, Q. Liu, M. Shi, W. Feng, F. Li, Lanthanide-Doped Nanoparticles with Upconversion and Downshifting Near-Infrared 
Luminescence for Bioimaging, Inorg. Chem. 2019, 58,14, 9351-9357. https://doi.org/10.1021/acs.inorgchem.9b01071

[21] M. Back, E. Trave, P. Riello, J. J. Joos, Insight into the Upconversion Luminescence of Highly Efficient Lanthanide-Doped $\mathrm{Bi}_{2} \mathrm{O}_{3}$ Nanoparticles, J. Phys. Chem. C 2018, 122, 13, 7389-7398. https://doi.org/10.1021/acs.jpcc.8b00637

[22] E. Ringe, J. Zhang, M. R. Langille, K. Sohn, C. M. Cobley, L. Au, Y. Xia, C. A. Mirkin, J. Huang, L. D. Marks and R. P. Van Duyne, Effect of Size, Shape, Composition, and Support Film on Localized Surface Plasmon Resonance Frequency: A Single Particle Approach Applied to Silver Bipyramids and Gold Nanocubes, Mater. Res. Soc. Symp. Proc., 2010, 1208, O10-02.

[23] C. L. Haynes, A. J. Haes and R. P. Van Duyne, Nanosphere Lithography: Synthesis and Application of Nanoparticles with Inherently Anisotropic Structures and Surface Chemistry, Mater. Res. Soc. Symp. Proc., 2001, 635, C6.3.1-C6.3.6.

[24] W. Haiss, N. T. K. Thanh, J. Aveyard and D. G. Fernig, Determination of size and concentration of gold nanoparticles from UVvis spectra, Anal. Chem., 2007, 79, 4215-4221. doi: 10.1021/ac0702084

[25] S. Peng, J. M. McMahon, G. C. Schatz, S. K. Gray and Y. Sun, Reversing the size-dependence of surface plasmon resonances, Proc. Natl. Acad. Sci. U. S. A., 2010, 107, 14530-14534. https://doi.org/10.1073/pnas.1007524107

[26] K. L. Kelly, E. Coronado, L. L. Zhao and G. C. Schatz, The Optical Properties of Metal Nanoparticles: The Influence of Size, Shape, and Dielectric Environment, J. Phys. Chem. B, 2003, 107, 668-677. https://doi.org/10.1021/jp026731y

[27] U. Kreibig, Electronic properties of small silver particles: the optical constants and their temperature dependence, J. Phys. F: Met. Phys., 1974, 4, 999. https://doi.org/10.1088/0305-4608/4/7/007

[28] U. Kreibig, ,J. Phys., 1977, 38, C2-C97.

[29] T. Linnert, P. Mulvaney and A. Henglein, Surface chemistry of colloidal silver: surface plasmon damping by chemisorbed iodide, hydrosulfide (SH-), and phenylthiolate, J. Phys. Chem., 1993, 97, 3, 679 682. https://doi.org/10.1021/j100105a024 
[30] M. D. Scanlon, P. Peljo and M. A. M'etraux, Charging and discharging at the nanoscale: Fermi level equilibration of metallic nanoparticles, Chem. Sci., 2015, 6, 2705. https://doi.org/10.1039/C5SC00461F

[31] W. Hou and S. B. Cronin, A Review of Surface Plasmon Resonance-Enhanced Photocatalysis, Adv. Funct. Mater., 2013, 23, 16121619. https://doi.org/10.1002/adfm.201202148

[32] Y. Zhang, A. Dragan and C. D. Geddes, Wavelength Dependence of Metal-Enhanced Fluorescence, J. Phys. Chem. C, 2009, 113, 12095. https://doi.org/10.1021/jp9005668

[33] J. R. Lakowicz, Radiative decay engineering 5: metal-enhanced fluorescence and plasmon emission, Anal. Biochem., 2005, 337(2), 171-94. doi: 10.1016/j.ab.2004.11.026.

[34] A. Bek, R. Jansen, M. Ringler, S. Mayilo, T. A. Klarm and J. Feldmann, Fluorescence Enhancement in Hot Spots of AFM-Designed Gold Nanoparticle Sandwiches, Nano Lett., 2008, 8, 485-490. https://doi.org/10.1021/n1072602n

[35] M. Ganguly, J. Pal, C. Mondal, A. Pal and T. Pal, Intriguing manipulation of metal-enhanced fluorescence for the detection of $\mathrm{Cu}(\mathrm{II})$ and cysteine, Chem.-Eur. J., 2014, 20, $12470 \quad$ - 12476. doi: $10.1002 /$ chem.201402505

[36] E. Susie and M. A. El-Sayed, Why gold nanoparticles are more precious than pretty gold: Noble metal surface plasmon resonance and its enhancement of the radiative and nonradiative properties of nanocrystals of different shapes, Chem. Soc. Rev., 2006, 35, 209. https://doi.org/10.1039/B514191E

[37] J. R. Lakowicz, Radiative Decay Engineering: Biophysical and Biomedical Applications, Analytical Biochemistry, Anal. Biochem., 2001, 298, 1. https://doi.org/10.1006/abio.2001.5377

[38] C. D. Geddes, H. Cao, I. Gryczynski, I. Z. Gryczynski, J. Fang and J. R. Lakowicz, Metal-Enhanced Fluorescence (MEF) Due to Silver Colloids on a Planar Surface: Potential Applications of Indocyanine Green to in Vivo Imagingt, J. Phys. Chem. A, 2003, 107, 3443. https://doi.org/10.1021/jp022040q

[39] K. Aslan, J. R. Lakowicz and C. D. Geddes, Rapid deposition of triangular silver nanoplates on planar surfaces: application to metalenhanced fluorescence, J. Phys. Chem. B, 2005, 109, 6247-6251. doi: $\underline{10.1021 / j p 044235 z}$ 
[40] K. Aslan, Z. Leonenko, Fast and Slow Deposition of Silver Nanorods on Planar Surfaces: Application to Metal-Enhanced Fluorescence, J. R. Lakowicz and C. D. Geddes, J. Phys. Chem. B, 2005, 109, 3157-3162. doi: 10.1021/jp045186t

[41] K. Aslan, M. Wu, J. R. Lakowicz and C. D. Geddes, Metal enhanced fluorescence solution-based sensing platform 2: fluorescent core-shell Ag@SiO2 nanoballs, J. Fluoresc., 2007, 17, 127-31. doi: 10.1007/s10895-007-0164-6

[42] H. Diehl, C. C. Hach and J. C. Bailar, Inorganic Syntheses, ed. L. F. Audrieth, John Wiley \& Sons, Inc., Hoboken, NJ, USA, 1950, vol. 3, doi: 10.1002/9780470132340.ch53.

[43] G. B. Roy, Synthesis and study of physico-chemical properties of a new chiral Schiff base ligand and its metal complex, Inorg. Chim. Acta, 2009, 362, 1709-1714. https://doi.org/10.1016/j.ica.2008.08.009

[44] G. D. Liu, J. P. Liao, S. S. Huang, G. L. Shen and R. Q. Yu, Fluorescence spectral study of interaction of water-soluble metal complexes of Schiff-base and DNA, Anal. Sci., 2001, 17, 1031. doi: 10.2116/analsci.17.1031.

[45] P. Chen, R. Lu, P. Xue, T. Xu, G. Chen and Y. Zhao, Emission Enhancement and Chromism in a Salen-Based Gel System, Langmuir, 2009, 25, 8395. https://doi.org/10.1021/la8035727

[46] M. L. Deda, M. Ghedini, I. Aiello and A. Grisolia, A New Blue Photoluminescent Salen-like Zinc Complex with Excellent Emission Quantum Yield, Chem. Lett., 2004, 33, 1060-1061. https://doi.org/10.1246/cl.2004.1060

[47] C. E. White and F. Cuttitta, Fluorometric Study of MagnesiumBissalicylidene-ethylenediamine System, Anal. Chem., 1959, 31, $2083-$ 2087. https://doi.org/10.1021/ac60156a064

[48] M. E. Germain and M. J. Knapp, Turn-on Fluorescence Detection of H2O2 and TATP, Inorg. Chem., 2008, 47, 9748-9750.

[49] J. H. Lee, A. R. Jeong, I. S. Shin, H. J. Kim and J. I. Hong, Fluorescence turn-on sensor for cyanide based on a cobalt(II)coumarinylsalen complex, Org. Lett., 2010, 12, 764. doi: $10.1021 / \mathrm{ol} 902852 \mathrm{~g}$

[50] I. Aoki, A. Takahashi and K. Watanabe, Determination of Aliphatic Primary Amines by Flow Injection Fluorometry Using 
Beryllium-Schiff Base Complexes, Anal. Sci., 1992, 8, 323-327. https://doi.org/10.2116/analsci.8.323

[51] K. Aslan, S. N. Malyn and C. D. Geddes, Metal-Enhanced Fluorescence from Gold Surfaces: Angular Dependent Emission, J. Fluoresc., 2007, 17, 7-13. https://doi.org/10.1007/s10895-006-0149-x

van Amerongen and V. Subramaniam, Silver Nanoparticle Aggregates as Highly Efficient Plasmonic Antennas for Fluorescence Enhancement, $J$. Phys. Chem. C, 2012, 116, 16687-16693. https://doi.org/10.1021/jp305720q

[53] Y. Zhang, K. Aslan, M. J. R. Previte and C. D. Geddes, Metalenhanced fluorescence from copper substrates, Appl. Phys. Lett., 2007, 90, 173116. https://doi.org/10.1063/1.2732185

[54] P. P. Pompa, L. Martiradonna, A. Della Torre, F. Della Sala, L. Manna, M. De Vittorio, F. Calabi, R. Cingolani and R. Rinaldi, Metalenhanced fluorescence of colloidal nanocrystals with nanoscale control, Nat. Nanotechnol., 2006, 1, 126. doi: 10.1038/nnano.2006.93

[55] E. Dulkeith, M. Ringler, T. A. Klar, J. Feldmann, J. A. Munoz and W. J. Parak, Gold Nanoparticles Quench Fluorescence by Phase Induced Radiative Rate Suppression, Nano Lett., 2005, 5, 585. https://doi.org/10.1021/n10480969

[56] M. Ganguly, A. Pal and T. Pal, Purification of Gold Organosol by Solid Reagent, J. Phys. Chem. C, 2012, 116, 9265-9273. https://doi.org/10.1021/jp2121356

[57] H. Mertens and A. Polman, Plasmon-enhanced erbium luminescence, Appl. Phys. Lett. 89, 211107 (2006); https://doi.org/10.1063/1.2392827.

[58] Y. S. Yamamoto, M. Ishikawa, Y. Ozaki and T. Itoh, Fundamental studies on enhancement and blinking mechanism of surfaceenhanced Raman scattering (SERS) and basic applications of SERS biological sensing, Frontiers of Physics, 2014, 9, 31-46. https://doi.org/10.1007/s11467-013-0347-3

[59] S. Sarkar, M. Pradhan, A. K. Sinha, M. Basu and T. Pal, Selective and Sensitive Recognition of $\mathrm{Cu} 2+$ in an Aqueous Medium: A SurfaceEnhanced Raman Scattering (SERS)-Based Analysis with a LowCost Raman Reporter, Chem.-Eur. J., 2012, 18, 6335-6342. http://dx.doi.org/10.1002/chem.201103458 
